\title{
La influencia que tiene el videojuego DOTA2 en el estado de ánimo del jugador aplicado al equipo "AMX"
}

The influence that the DOTA2 videogame has on the mood of the player applied to the "AMX" team

Paulina Fernanda Mora Piña. ${ }^{1}$, Vanessa Lorena Valverde González. ${ }^{2}$ \& Sofía Paulina Valle Oñate. ${ }^{3}$

\begin{abstract}
DOI: https://doi.org/10.33262/cienciadigital.v4i1.1078

Research to determine the influence of the Dota 2 video games on the mood of the players, an application research was used with the analytical method that seeks to find answers to a problem, produce contributions that promote the field of medicine and the computer science and new meanings to the current forms of the influence exerted by video games on the emotional health of people, responds to the analysis of results, the techniques applied are observation, surveys and interviews. In this work we will select the "AMX" team, DOTA2 players through surveys. The results analyzed after the different studies showed that the change of a normal behavior to another aggressive behavior of the players after losing a game in specific ways increasing their irritability and tension, on the other hand, each member was visualized with a behavior more compulsive before the different actions that were presented in the games played.

Dota2 will be researched as a videogame, how it develops, how complicated it is to play and the need felt by players to spend more time each day.

The influence exerted by the video game Dota 2 on the changes in the mood of the people is analyzed due to the tension generated during each of the games, in addition to the violence that it maintains, the moods will be analyzed before start the game and after him.
\end{abstract}

Keywords: Dota2, Aggressiveness, Conduct, Videogames, Games.

\section{Resumen}

Los videojuegos se han convertido en una fuente muy rentable, existiendo gran variedad de ellos, Dota 2 es un videojuego muy conocido que afecta al estado de ánimo de los jugadores, para conocer los problemas médicos que causa la exposición de varias horas a esta actividad,

\footnotetext{
${ }^{1}$ Espoch, Unidad de Admisión y Nivelación, Riobamba, Ecuador, pmora@espoch.edu.ec

${ }^{2}$ Espoch, Mecánica, Riobamba, Ecuador, vanessa.valverde@espoch.edu.ec

${ }^{3}$ Espoch, Unidad de Admisión y Nivelación, Riobamba, Ecuador, paulina.valle@espoch.edu.ec
} 
se ha utilizado una investigación aplicativa con el método analítico que busca encontrar respuestas a un problema, para producir aportes que impulsen el campo de la medicina y de la informática. La influencia que ejercen los videojuegos sobre la salud emocional de las personas responde al análisis de resultados, las técnicas aplicadas como son la observación, encuestas y entrevistas. El presente trabajo se basó en el equipo "AMX", jugadores de DOTA2, los resultados analizados manifestaron el cambio de una conducta pacífica a otra agresiva de los participantes luego de perder un juego de formas especificas aumentando su irritabilidad y tensión, por otra parte, se visualizó a cada uno de los integrantes con un comportamiento más compulsivo ante las diferentes acciones que se presentaron en las partidas jugadas.

Dota 2 como videojuego, tiene algunos niveles que cada jugador debe cruzar para llegar a la meta, aumentando la dificultad a medida que avanza, de tal manera que cada jugador siente la necesidad de dedicarle más tiempo por día, generando un cambio de ánimo, violencia y estrés en cada partida, por lo tanto, se realizará un estudio del cambio que experimenta el jugador antes y después de una partida, ganada o perdida y los daños que se han producido a nivel de nervios y habilidades del jugador.

Palabras claves: Dota2, Agresividad, Conducta, Videojuegos, Partidas.

\section{Introducción}

En los últimos años el estudio del estado de ánimo de las personas en cuanto al desarrollo de cierta actividad ha crecido desde el punto de vista Psicológico. Así, desde los años 80 la base de datos de la revista PsycLIT de la APA recoge un sin número de investigaciones orientadas con el estado de ánimo de las personas y en algunos casos la influencia que los videojuegos implican debido al estrés que generan (Sanz, 2001).

El cerebro es uno de los órganos más importantes del cuerpo humano, el uso desmedido de los videojuegos por lapsos de más de dos horas y explosión a los destellos de la pantalla puede generar daños significativos. La salud es uno de los elementos más relevantes para el desarrollo de una vida larga y cualitativa (Bembibre, 2011).

Cuando se habla de estrés y cambios en el estado de ánimo debido al uso inadecuado de un videojuego, se habla de daños emocionales y físicos producidos por la tensión que ejerce. Mientras que los creadores de los videojuegos han mencionado que están hechos para divertir y sacar del aburrimiento o del estrés post - trabajo u estudio a las personas, no para producir en ellos un malestar emocional.

Los jugadores obsesivos siempre han existido, esta es una problemática donde cada jugador vive su propia novela el momento que está dentro del juego (Pérez \& Bedoya, 2007). La adicción a este tipo de videojuegos, en este caso DOTA2, puede conllevar a grandes cambios psicológicos debido a que los jugadores invierten gran parte de su tiempo en un mundo imaginario, y olvidan por completo todo su entorno, la agresividad es una constante habitual ya que al estar frente a un monitor sienten el libre albedrio de agredir verbal y psicológicamente a los demás; además de otros problemas, esto con lleva a que el jugador se aleje de su vida social (Balzabal \& Rodríguez, 2012). 
De aquí que el tema es importante debido a la gran cantidad de videojuegos que se han desarrollado y el efecto que estos producen sobre el estado de ánimo de la persona. Este estudio del cambio de estado de ánimo de las personas al momento de jugar a un video juego en específico, tiene como objetivo crear conciencia de cómo afecta el uso diario y continuo de estos videojuegos, de hecho la gran cantidad de adolescentes buscan un método de pasar el tiempo y distraerse, sin embargo se debe tener en cuenta que este tipo de diversión momentánea puede causar daño en la expresión de nuestras actitudes y la forma de comportarnos con los demás, debido a la gran influencia que tienen en nuestra vida diaria (Etxeberria, 1998).

Se ha considerado al equipo AMX, el mismo que consta de un grupo de personas a quienes se les ha identificado con este nombre, y que han decidido involucrarse con el videojuego DOTA 2 para el desarrollo de la presente investigación. Siendo DOTA 2 un juego desarrollado por VALVE, que pertenece a la categoría de juegos ARTS (estrategia de acción en tiempo real), el mismo que es desafiante en el que se pone a prueba conocimientos, habilidades y destrezas, contra miles de jugadores de todo el mundo que están simultáneamente conectados.

El objetivo del presente trabajo es establecer influencia que tiene el videojuego DOTA2 en el estado de ánimo del jugador aplicado al equipo "AMX” desarrollado específicamente para comprobar los cambios de ánimo de una persona antes y después del uso del videojuego.

\section{Marco Teórico}

\section{Los Videojuegos}

Un videojuego es una aplicación interactiva orientada al entretenimiento que, a través de ciertos mandos o controles, permite simular experiencias en la pantalla de un televisor, una computadora u otro dispositivo electrónico (Porto \& Gardey, 2013).

Los videojuegos se diferencian de otras formas de entretenimiento por la manera en que deben involucrarse con los videojuegos al utilizar un mando como una palanca o teclas para desarrollar el juego.

Los videojuegos recrean entornos y situaciones virtuales en los que el video jugador puede controlar a uno o varios personajes (o cualquier otro elemento de dicho entorno), para conseguir uno o varios objetivos por medio de reglas determinadas (EcuRed, 2010).

Los Gamers al grupo de jugadores experimentados, que se caracterizan por llevar varios años jugando, invertir bastantes horas de juego y tener una gran diversidad en sus gustos, en cuanto a géneros y tipos de plataformas. Además, es un consumidor asiduo que le gusta estar bien informado en cuanto a los productos que ofrece el mercado, sus características y nuevas tendencias. Los videojuegos tienen varias características como: son actividades con fines recreativos y en algunos casos instrumentos educacionales, promueven la estimulación mental o física, ayudan en el desarrollo de habilidades prácticas y sociales, logra que los jugadores tengan experiencias diferentes a las reales (EcuRed, 2010).

Los videojuegos no son tan malos como se piensa. Pueden estimular la creatividad y no necesariamente promover el sedentarismo (Espinoza, 2016). 
De acuerdo con Andrés Hoyos Melo, psicólogo educativo de la Universidad Nacional de Colombia, "los videojuegos son un desarrollo tecnológico acorde a nuestros tiempos; se basan en principios de competencia y están directamente relacionados con el uso de estrategia, agilidad y concentración (Flores \& Capa, Research Gate). Los videojuegos pueden ser utilizados como herramientas educativas, en la medida en que su uso no difiere mucho de lo que se hace o podría hacerse con las nuevas tecnologías de la información".

Sin embargo, Andrés Hoyos Melo, psicólogo educativo de la Universidad Nacional de Colombia advierte que: "es importante tener en cuenta que el videojuego como herramienta educativa, por sí solo, no funcionaría si no hay una orientación con fines pedagógicos por parte de un mediador (docente o facilitador), ni se tiene claro el mensaje o el tipo de aprendizaje que con él se quiere motivar"

Los Deportes Electrónicos o eSports son competiciones de videojuegos multijugador que se han convertido en eventos de gran popularidad. Los géneros más comunes en los videojuegos asociados a los eSports son: estrategia en tiempo real, disparos en primera persona y arenas de batalla multijugador online (RedUsers, 2019).

\section{Problema de los video juegos}

Es necesario tener en cuenta de la manera de crecimiento de los videojuegos en actividades educativas y formativas, también utilizado en gran cantidad de terapias (Pinos, 2009), por este motivo es necesario un análisis profundo de cara al futuro en la utilización positiva de este mecanismo moderno, los videojuegos se realizan en un ambiente social el cual puede ser violento, ya que vinculan a los jugadores en una escenografía imaginaria, como por ejemplo DOTA2 el temática del juego consiste en intentar matar a tus enemigos con la finalidad de terminar siendo el más fuerte para así ganar la partida.

Este tipo de videojuegos, denominados videojuegos violentos, producen en los jugadores una conducta más agresiva, por este motivo no son aptos para todas las edades, generando una restricción para su libre circulación, la misma que por parte de las autoridades y organismos no ha sido verificada, debido a que los desarrolladores de los diferentes tipos de videojuegos no analizan el tipo de público al que va destinado, antes de sacarlo a la venta en sus plataformas de libre acceso. Sin embargo, en diferentes partes del mundo como California, el parlamento Suizo, prohibieron el uso de videojuegos los cuales incitaban a la violencia a los niños y adolescentes (Chamorro, Carbonell, Manresa, Muñoz, \& Ortega, 2014).

Algunos científicos realizaron diferentes tipos de investigaciones como C. Anderson, quien investigó acerca del campo de la influencia de los videojuegos en la conducta, empezó analizando el aumento diario en el uso de videojuegos de jóvenes aproximadamente de 4-16 horas por semana y que los preferidos eran los de violencia, este tipo de videojuegos incitan a matar personas ya sean inocentes o culpables por este motivo el jugador asume el papel de un héroe aunque otros casos puede ser el criminal sin darse cuenta, los resultados arrojaron que la conducta agresiva presentada se relacionaba con estadísticamente con los videojuegos, esto se debe a la desestabilización emocional que puede provocar así como la falta de empatía y falta de conducta 
prosociales, también se pudo demostrar que son un factor causal de riesgo a largo plazo (Martí, 1994).

Otro grupo de expertos, llegaron a la conclusión de que los videojuegos generan la agresividad entre quienes lo utilizan, tanto en el momento en que se está jugando, como posteriormente (Vivas, 2000). Demostrando que este tipo de videojuegos puede promover agresividad en las personas ya que los jugadores toman un papel activo en su mundo creado en el videojuego, y realiza las acciones por él mismo, además se le debe añadir las nuevas tecnologías que se han desarrollado lo que permite experimentar un ambiente más real en el videojuego.

\section{Deporte Electrónico}

Deporte es una palabra que significaba ejercicio, gimnasio, sudor, entrenamiento, aire libre, los cuales aportan un valor significativo a nuestro desarrollo físico y mental.

Ahora se habla también de los eSports o deportes electrónicos que son los videojuegos y se practica desde la comodidad de su casa.

Bajo un estudio realizado el $51 \%$ de los votantes consideran que los eSports deberían considerarse solo como un videojuego, el $27 \%$ no conoce aún de la existencia de los deportes electrónicos y solo el 22\% considera que los eSports son como un deporte más (Alarcón, s.f.)

Desde el punto de vista de las personas participantes, se cree que los videojuegos no deben ser considerados como un deporte, debido a que la definición de deporte habla de un bienestar corporal y mental que ayude a refrescar el estrés diario causado por el trabajo, estudio o por las diferentes actividades que se realiza durante el día.

\section{Dota 2}

Con el pasar de los días y el tiempo que las personas lo dedican a jugar se va convirtiendo en una pasión. Hoy en día en muchos países se realizan tornos de este video juego, ahora llamados los DEPORTES ELECTRÓNICOS.

Dota2 es un videojuego de estrategia en tiempo real multijugador en línea entregado por la plataforma Steam con su subdivisión Valve presentado en la blizzCon de 2005 (Mirzadeh, Kumar, \& Gansow, 1993).

Son considerados el fenómeno de la comunidad Gamer llamados los juegos MOBA (Multiplayer Online Battle Arena) o DOTA 2 (Defense of the Ancients) necesita de gran comunicación y trabajo en equipo para lograr la victoria, además la variedad de héroes la complejidad de las (Sánchez \& Torres, 2014) habilidades e infinidad de estrategias aseguran cada una de las partidas como únicas. La temática del juego es escoger un héroe de 113 existentes, cada uno tiene habilidades y debilidades diferentes de los demás, también estos poseen atributos específicos que pueden combinarse con objetos que se consiguen dentro de la partida con el fin de potenciar sus poderes, además se juega en equipos que pueden ser aleatorios o elegir con quien jugar dando como resultado un enfrentamiento de 5v5 con el objetivo de destruir una "estructura principal" del equipo contrario, teniendo que atravesar varias líneas de defensa y a los héroes adversarios que van a 
impedir la llegada del jugador a dicha construcción , el mapa es el mismo para todas las partidas, así pues se necesita una habilidad estratégica, reflejos, trabajar bien bajo presión, etc. (Christoph Eggert, 2015).

Dota 2 está catalogado como un juego de estrategia, momento y acción, su característica principal es que es desarrollado mediante órdenes y objetos y es de modalidad Juego de guerra. Se forman dos equipos que se enfrentan en un vasto campo de batalla dividido en 2 partes, donde el primero en destruir el territorio del oponente llamado gana.

Pero el factor más determinante es el trabajo en equipo debido a la coordinación que se necesita para alcanzar la victoria, el problema surge en el momento de la comunicación con los demás jugadores ya que el anonimato que nos proporciona el internet permite realizar abusos verbales y psicológicos por parte de un jugador hacia el otro, sumando el estrés que se presenta debido a la gran concentración que requiere el juego, induce en el usuario un cambio emocional drástico de una manera acelerada, los cambios más usuales que presentan dentro del videojuego así como en su entorno social o el ambiente que está presente el individuo con sus allegados son la agresividad, irritabilidad, odio, discriminación, entre otras.-

\section{Metodología}

En el diseño de la investigación se utilizó el método cuasi experimental analizando todas las observaciones efectuadas en circunstancias reales, sin afectar ni perjudicar a los participantes. Siendo base el método analítico, con un enfoque Cualitativo, que permite conocer el objeto de estudio, con lo cual se puede: explicar, hacer analogías, comprender mejor su comportamiento y establecer nuevas teorías.

Las variables son el cambio de humor, el estrés, el trabajo en equipo y las relaciones interpersonales que están vinculadas al cambio de ánimo de una persona sea este jugador o no. Esta investigación se ha llevado a cabo en el equipo de Dota 2 "AMX" provenientes de la ciudad de Riobamba-Ecuador para conocer si existen cambios en sus estados de ánimo, los datos utilizados en este estudio proceden de una muestra de 10 sujetos ( 7 hombres y 3 mujeres).

Una vez delineada la idea de esta propuesta, se inicia con un análisis de bibliografía, a nivel de cambios de ánimo y estrés en los jugadores de los juegos de video en general, dando como resultado datos generales y no específicos como es el de este caso, razón por la cual el interés por el tema creció determinando así un estudio descriptivo debido a la no existencia de mucho material referencial y hallazgos, lo que permitirá que se aborde el tema en todo tipo de personas.

Constituye una propuesta novedosa en el campo profesional que sigue las siguientes pautas:

- La fundamentación teórica.

- El análisis de un videojuego.

- El resultado o producto del trabajo.

Además, responde al análisis de los resultados, de las encuestas realizadas antes y después del uso del videojuego, por lo que se debe aclarar que los datos que se obtengan no serán generalizados ni representaran a toda una población de jugadores, pero si servirán como base de estudio.

Las técnicas aplicadas son observaciones, encuestas, asistencia a participantes y entrevistas. 
La obtención de información se la realizó por medio de encuestas con preguntas abiertas, el análisis de los resultados se hicieron por medio de una comparación, entre una encuesta inicial antes de exponer a los participantes a varias partidas de dota2 y una encuesta final, después de varias partidas ganadas y perdidas, con el fin de verificar si existía un cambio que sirva para explicar la hipótesis de este estudio, todo este protocolo utilizado tuvo una duración de 2 semanas aproximadamente, sin influir de ninguna manera en las partidas jugadas por los individuos.

\section{Resultados}

Se procede a la tabulación de los datos adquiridos en las encuestas aplicadas.

\section{Estrés bajo presión}

En la mayor parte de personas el trabajo bajo presión aumenta el cansancio en las personas y genera estrés por lo tanto disminuye el rendimiento en las actividades.

A partir de lo antes expuesto se ha tomado en cuenta que cada uno de los video jugadores tiene actividades que desarrollan durante su vida cotidiana, siendo estudiantes universitarios y empleados; por lo que lo deben tener una total concentración en lo que hacen. Como primer caso se analizó al grupo que, si le gusta trabajar bajo presión, observando las actividades que realizan normalmente y dependiendo de las horas que le dedican a juego se puede decir que:

Tanto empleados como estudiantes les gustaría combinar su tiempo entre las actividades diarias y el juego buscando diversión o una manera de desestrés.

En el segundo caso las personas encuestadas prefieren vivir o trabajar sin presión, libertad propia en el desarrollo de sus actividades y evitar el estrés.

\section{Cambios de humor con facilidad}

El humor es una característica de todo ser humano, por lo tanto, cuando se desarrolla cualquier actividad bajo presión, el cuerpo experimenta cierta cantidad de estrés lo que implica cambios inmediatos de corto y largo plazo en el humo de la persona.

Dentro del grupo de personas encuestadas, se encontró un grupo que no cambian de humor fácilmente, debido a la manera en que desarrollan sus actividades poniendo tiempos en cada una de ellas.

\section{Demuestra toxicidad ante errores de otras personas}

Una persona tóxica es aquella que constantemente altera a las personas que están a su alrededor, haciendo imposible su bienestar.

Después de conversar con los participantes, las dos personas que dicen ser tóxicas hablan de que les gusta ser el centro de las cosas y les molesta las equivocaciones y se tornan insistentes hacia las personas afectadas.

Los demás participantes hablan de que la autoestima de las personas y su humor influyen mucho en el desarrollo de sus actividades por ese motivo tratan de ayudar a relajar a las personas cuando cometen equivocaciones para que disfruten de lo que hacen sin perjudicar su autoestima. 


\section{Existe comodidad trabajando en equipo}

Según Ransdtad en el año 2016 el trabajo en equipo se define como la unión de dos o más personas organizadas de una forma determinada, las cuales cooperan para lograr un fin común que es la ejecución de un proyecto.

Se pudo percibir que las personas dijeron que si se relacionan complementariamente en el entorno en el cual se desarrollan buscando alcanzar los retos propuestos que no se lograrían individualmente.

Hablan también de aunar aptitudes que potencializaran esfuerzos disminuyendo tiempo y aumentando la eficacia y eficiencia.

Además de fomentar la coordinación, el compañerismo, la comunicación el compromiso y la confianza.

\section{Mantiene buena comunicación interpersonal}

La comunicación entre un grupo de personas trata los lazos directos que relacionan las personalidades. Zaldívar plantea que "La comunicación interpersonal puede ser considerada como el proceso de intercambios de mensajes entre dos o más personas, con la finalidad de alcanzar determinados objetivos". La personalidad es el escondrijo en que se haya la esencia de la comunicación. La comunicación es la base para la construcción de la personalidad.

Una buena comunicación se da a partir de un buen trabajo en equipo siendo lo suficientemente tolerantes creando confianza entre las personas, lo que permitirá una buena relación con los demás, brinda confianza y permite una participación activa de todos los miembros.

En fin, se observó que los resultados de la primera encuesta realizada representada evidenciaron datos en los cuales la gran mayoría de encuestados no presentaba cambios de humor refiriendo las variables que se habían propuesto en cada pregunta ya que estas hacían alusión a los problemas que se generan al momento de jugar una partida online ya que en la realización del juego se presume que pueden presentar cambios bruscos de presión, humor y estrés.

Se procede entonces a analizar los datos post partida, después de haber jugado las 5 partidas que se propuso para la realización del presente estudio.

\section{Disfruta de las partidas}

Una vez concluidas las partidas, el grupo de 6 personas dijeron que si habían disfrutado estas partidas ya que les gusta sentir esa presión que el juego les brinda y que les gustaría seguir jugando, debido a que están desarrollando estrategias de juego muy valiosas y se quedan con la ansiedad de seguir jugando.

Las personas que dijeron que no, hablan de que es un juego de mucha violencia.

\section{Estrés en algún momento de la partida}

En el desarrollo de las partidas se puedo encontrar un grupo de personas a las que en algunos instantes sintieron máxima tensión durante el juego por lo que les ha provocado dolores intensos de cabeza, frustración y reniego durante el juego y aún más si es el juego en grupo donde se van las palabras y hasta se llega a herir susceptibilidades. 
Además, se determinó que los videojuegos son para divertirse y desestresarse, lo que no se pudo lograr con Dota 2, no se debe convertir en una adicción y mucho menos en obsesión. Notándose además de que el hecho de competir hace que quieran seguir inmersos en el juego, exigiéndose más de lo que pueden dar sin darse cuenta el daño que producen a su salud, su cuerpo y a su mente. Mientras que las personas que no se estresaban, era debido a que jugaban en horarios establecidos y por un tiempo limitado tratando solo de desestresarse. Y si llega el punto donde lo único que consigo es estrés lo deja para estabilizarse emocionalmente.

\section{Cambio de humor durante la partida}

Una persona estresada es una persona que no tiene tranquilidad que vive desesperaciones y por lo tanto un cambio de humor consigo mismo y con las personas que lo rodean.

Entre más horas le inviertas al video juego más son los factores que van a influir en la persona como prominencia, intolerancia, apartamiento del entorno, conflictos con quien los rodea, causando un deterioro en sus relaciones personales o desatender sus responsabilidades

\section{La suma de los resultados de las partidas influyó en su vida diaria}

Hubo un cambio muy brusco en las respuestas que se relacionaban con este trabajo de investigación, así se evidencian en la encuesta 2, en donde la mayoría de encuestados demostraron que, si hay cambios de humor que pueden ser diferentes en cada persona, pero normalmente se trataba de irritabilidad, agresión y un cambio en las emociones que normalmente poseen estas personas.

Después de analizadas las encuestas a los video jugadores se les hizo una última pregunta informal obteniendo los siguientes resultados:

\section{Horas de uso}

Al realizar el análisis de los resultados se determinó que el juego DOTA 2, genera una adicción de tal manera que los jugadores que estuvieron expuestos de 1 a 2 horas creen que es una bonita experiencia les permite entretenerse; los video jugadores que lo hacen de 3 a 4 horas diarias comentan que es una experiencia única, les gusta mucho el juego y más aún cuando avanzan en las etapas; los video jugadores de entre 4-5 horas hablan de que su vida cambio por completo con el uso de este videojuego que es una experiencia única que no les gustaría que se acabe.

Según lo analizado anteriormente, se observó que las personas que juegan entre 1 a 2 horas no sufrieron de un cambio en su estado de ánimo, tampoco sintieron la necesidad de seguir jugando, mientras que con los jugadores expuestos de 2 a 3 horas se observó un nivel bajo de ansiedad por querer seguir jugando, considerando que estos jugadores están conscientes de que tienen responsabilidades que cumplir.

Los jugadores que utilizaron de 4 a 5 horas hablan mucho del juego incluso dentro de sus actividades diarias, existe un pequeño desinterés para con las personas que los rodea. Generando 
un cambio de humor más aun cuando por cumplir con sus responsabilidades no pueden continuar con el juego.

Dota 2 ha demostrado ser un juego muy entretenido para quienes les gusta los juegos de armas, muerte y violencia. Creando tensión, agresividad en los jugadores que hacen uso de este. Sin embargo, los creadores del juego dota2 han creado torneos en EE. UU., en donde la afluencia del público ha sido extensa, esto incita a más personas a estar dentro de este mundo cibernético y dejar de lado la realidad en la que se encuentran.

\section{Discusión}

La influencia que tienen los videojuegos Dota 2 en el estado de ánimo de los jugadores resulta en estrés. Existen estudios que hablan de un amplio crecimiento en popularidad de los Juegos Dota 2 pero ningún habla de los efectos que causa en los jugadores. Antes de iniciar las partidas online los individuos presentaban una actitud relajada y tranquila, lo que cambio después de terminar el juego, presentaron estrés por tratar de ganar a sus contrincantes, esto a su vez desencadeno un cambio de humor negativo entre ellos y consigo mismos llegando al punto de reproches e insultos al momento de errar en alguna parte de las partidas que se jugaron.

Al hablar de los hallazgos se puede decir que el estrés es el factor más influyente en los cambios de ánimo de los jugadores sin importar la edad y el sexo debido a que el juego dota 2 es de mucha violencia más aun en su práctica no controlada.

Los juegos de video deben ser utilizados para evitar el estrés mental, deben ser utilizados durante un tiempo corto y determinado del día, ya que el uso continuo y por largas jornadas de tiempo tiende a provocar un estrés causado por la presión que ejerce la violencia definida en Dota 2, lo que implica un cambio en el estado de ánimo por el efecto que tiene la violencia en nuestro cerebro. Por último, al terminar y retirarse cada uno, expresaron en su medio social y con las personas que convivían diariamente una actitud poco normal con la manifestación más común entre ellos que fue la irritabilidad, por el simple hecho de haber tenido una partida mala de todas las que jugaron o por errores cometidos por sus compañeros que pudieron comprometer la victoria del equipo.

Tomando en cuenta las encuestas básicamente en la pregunta 1 y 2 las cuales hacen referencia a el estrés que les provoca antes y después del uso del videojuego y al cambio de humor que esto provoca ser puede decir que:

\section{Representación comparativa antes y después de una partida}

El nivel de estrés en los jugadores es el factor predominante en esta investigación por lo tanto se genera un cambio inminente el humor del jugador.

De acuerdo con nuestros datos antes del aplicar el juego las personas encuestadas presentan un nivel de estrés del $60 \%$ en sus actividades diarias, después de haber experimentado el juego el nivel de estrés es de un 90\%. Según entrevistas a los jugadores, el nivel de estrés se da por la presión que ejerce el video juego en cada uno de ellos, ya que hablan de que cuando están jugando sienten que son parte de cada una de las etapas de videojuego.

Un aspecto importante que hay que tomar en cuenta es que los jugadores más activos son aquellos que permanecen más tiempo encerrados en sus casas, lo que indica que el hogar se ha convertido 
en lugar de riesgo por el comportamiento que se observa y que el control de cada jugador debe ser clave en este aspecto. Es primordial el control en el uso de los videojuegos por los padres, esposo o esposa o simplemente el jugador tiene que ejercer un control en el mismo en cuanto a las horas que dedica al video juego previniendo el riesgo de desarrollar adicción

Por otro lado, cuando se analiza el uso del videojuego y sus relación con otras variables predictoras, se halla que existe una analogía moderada entre el uso del videojuegos y las variables relacionadas como la toxicidad frente a errores cometidos, el trabajo en equipo y las relaciones interpersonales, a más de lo observado como la organización familiar, toma de decisiones en sus actividades cotidianas y comportamiento agresivo en donde, se pudo observar que hay una relación contradictoria entre la organización familiar y el video juego, a menor interés familiar mayor uso, así como a menor toma de decisiones adecuadas mayor uso de los videojuegos.

Se recomienda que el uso de los videojuegos en los jugadores debe ser normado tanto por los padres como hermanos, amigos y sociedad en general, estableciendo límites para el desarrollo favorable de otras actividades, lo importante es entretenerse, desestresarse no producir una adicción por el uso no controlado de este videojuego.

\section{Conclusiones}

El uso de los videojuegos ayuda a salir de la depresión, cuando se cree que todo está perdido, hace mejorar el humor y las ganas de seguir adelante. Si bien no todas las personas tienen la misma capacidad de realizar actividades bajo circunstancias hostiles, manteniendo la eficiencia; el trabajo bajo presión es una habilidad que se puede aprender.

El consumo propasado de videojuegos produce un cambio psicológico ya que muestra un ambiente violento y esto puede ser el inicio para debatir los diferentes tipos de problemas causados por el uso excesivo de videojuegos, lo que conllevaría a conflictos sociales, morales y violencia con las personas que nos rodean.

Se observó que existe un cambio de comportamiento en los participantes después de haber jugado durante varias partidas Dota2, con el hecho de tener como objetivo matar para poder ganar crea un ambiente propicio para producir ansiedad en el jugador, esto dificulta su manejo de la agresión, finalmente la adicción a este videojuego es muy grave, debido a que, a mayor frecuencia de uso, los cambios psicológicos son negativos.

Hay que tomar en cuenta también que Dota 2 cuenta con una variedad de héroes, habilidades y poderosos objetos, lo que conlleva a que sus usuarios creen que Dota 2 no tiene fin. Dota 2 no pone límites a la manera que el participante juega, da el poder de expresar su propio estilo a su jugador lo que implica que entre más juegue una persona más lo va querer seguir haciendo, lo que implica que se va a convertir en un círculo vicioso que si no se controla su uso siempre habrá problemas.

\section{Referencias bibliográficas}

Alarcón, J. O. (s.f.). Rincón de la Tecnología. Obtenido de Rincón de la Tecnología: https://rincondelatecnologia.com/esports-deporte-influencia/ 
Balerdi, F. E. (15 de 10 de 2017). Usal.es. Obtenido de Usal.es: https://campus.usal.es/ teoriaeducacion/rev_numero_02/n2_art_etxeberria.htm

Balzabal, L. T., \& Rodríguez, J. M. (2012). El papel de la educación en el tratamiento de la adicción a la taecnología. Hekademos, 12.

Bembibre, C. (04 de 23 de 2011). Importancia.org. Obtenido de Importancia. org: https://www.importancia.org/importancia-de-la-salud.php

Chamorro, A., Carbonell, X., Manresa, J., Muñoz, R., \& Ortega, R. (2014). The Questionnaire of Experiences Associated with Video Games (Cerv): An Instrument to Detect the Problematic Use of Video Games in Spanish Adolescents. Adicciones, 20.

Christoph Eggert, M. H. (2015). Classification of Player Roles in the Team-BasedMulti-player Game Dota 2. HAL, 15.

Flores, M. V., \& Capa, W. (Research Gate). Video Juegos: Adicción y Factores predictores. Jour, 9.

Mirzadeh, S., Kumar, K., \& Gansow, O. (1993). The Chemical Fate of 212Bi-DOTA Formed by $\beta$ - Decay of 212Pb(DOTA)2-***. Radiochimica Acta, 15.

Pérez, J. A., \& Bedoya, V. H. (2007). El juego de azar y el videojuego en la Universidad de San Buenaventura - Medellín. Grupo de Investigación de Estudios Psicosociales en Adicciones, GIEPSA, 10.

Sánchez, R. P., \& Torres, D. (2014). Intensidad de demanda de los videojuegos y su efecto sobre el estado de ánimo y la activación percibida. Universidad Psychol, 14.

Espinoza, R. C. (2016). Juegos digitales y gamificación aplicados en el ámbito de la educación. Revista Iberoamericana de Educación a Distancia, 7.

Etxeberria, X. (1998). Videojuegos y educación. Comunicar, 11.

Fragua, C. (30 de 8 de 2016). ORH Grupo Editorial de Conocimiento y Gestión, S.L. Obtenido de ORH Grupo Editorial de Conocimiento y Gestión, S.L.: https://www.observatoriorh.com/productividad/trabajar-bajo-presion-una-habilidadprofesional-cada-vez-mas-demandada.html

Funk, J. (1993). Reevaluating the Impact of Video Games. Sage Journals, 15.

Lynes, D. (10 de 01 de 2017). TeensHealth. Obtenido de TeensHealth: https://kidshealth.org/es/teens/stress-esp.html

Martí, J. E. (1994). Videojuegos, personalidad y conducta. Psicothema, 10. 
Padovani, A. (08 de 06 de 2018). Natursan. Obtenido de Natursan: https://www.natursan.net/que-es-una-persona-toxica-y-como-identificarla/

Pinos, C. C. (2009). Aspectos psicológicos del juego comercial. Tratamientos y programas preventivos. Hacia el juego responsable. Revista Docencia e Investigación, 34.

Porto, J. P., \& Gardey, A. (10 de 1 de 2013). Definiciones. Obtenido de Definición. DE: https://definicion.de/videojuego/

Ransdtad. (14 de 03 de 2016). El trabajo en equipo, la unión conlleva al éxito. Obtenido de Randstad: https://www.randstad.es/tendencias360/trabajo-en-equipo-la-union-conllevaal-exito/

RedUsers. (31 de 10 de 2019). RedUsers. Obtenido de RedUsers Comunidad de Tecnología: http://www.redusers.com/noticias/fenomenal-crecimiento-los-esports-latinoamerica/

Rodríguez, J. V. (2015). Las relaciones interpersonales componente esencial en el clima sociopsicológico de gimnasia rítmica: Una revisión Necesaria. OLIMPIA, Revista de la Facultad de Cultura Física de Granma, 11.

Sanz, J. (2001). Un instrumento para evaluar la eficacia de los procedimientos de inducción de estado de ánimo la EVEA. Análisis y Modificación de Conducta, 40.

Vivas, F. M. (2000). Adolescencia y Agresividad. Madrid: Universidad Complutense.

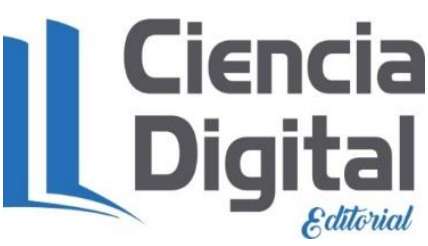




\section{PARA CITAR EL ARTÍCULO INDEXADO}

Mora Piña, P. F., Valverde González, V. L., \& Valle Oñate, S. P. (2020). La influencia que tiene el videojuego DOTA2 en el estado de ánimo del jugador aplicado al equipo "AMX" . Ciencia Digital, 4(1), 142-155. https://doi.org/10.33262/cienciadigital.v4i1.1078

\section{Ciencia}

El artículo que se publica es de exclusiva responsabilidad de los autores y no necesariamente reflejan el pensamiento de la Revista Ciencia Digital.

El artículo queda en propiedad de la revista y, por tanto, su publicación parcial y/o total en otro medio tiene que ser autorizado por el director de la Revista Ciencia Digital.
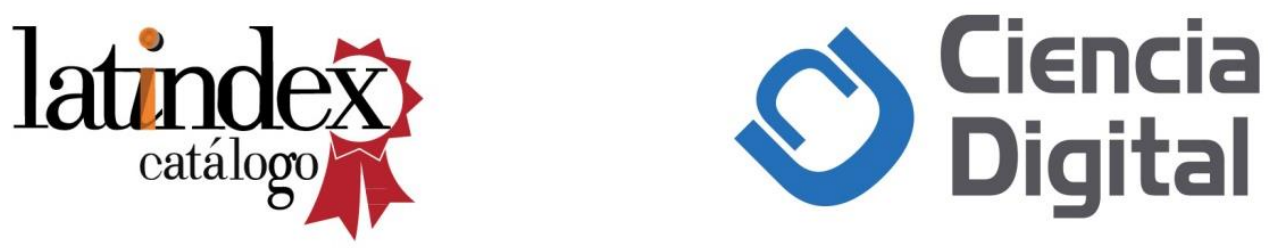